\section{Original Article}

Check for updates

\section{OPEN ACCESS}

Received: Jan 18, 2019

Revised: Feb 25, 2019

Accepted: Feb 27, 2019

Corresponding author:

Mi-Hae Sung

Department of Nursing, Inje University College of Medicine, Institute of Health Science, 75

bokji-ro, Busanjin-gu, Busan, Korea.

Tel: +82-51-890-6825

Fax: +82-51-896-9840

E-mail: nursmh@inje.ac.kr

(c) 2019 Korean Society of Women Health

Nursing

This is an open access article distributed under the terms of the Creative Commons Attribution Non-Commercial License (https:// creativecommons.org/licenses/by-nc/4.0/) which permits unrestricted non-commercial use, distribution, and reproduction in any medium, provided the original work is properly cited.

ORCID iDs

Eun Jung Lee iD

https://orcid.org/0000-0002-7294-1219

Mi-Hae Sung (iD)

https://orcid.org/0000-0002-5769-5857

Hye-Kyong Ahn (iD)

https://orcid.org/0000-0002-4214-9300

Yun Ah Kim iD

https://orcid.org/0000-0003-0295-0627

\section{Conflict of Interest}

The authors declared no conflict of interest.

\section{Author Contributions}

Conceptualization: Lee EJ, Sung MH, Ahn HK, Kim YA; Data curation: Lee EJ, Ahn HK; Formal analysis: Lee EJ, Ahn HK; Investigation Lee EJ, Ahn HK; Methodology: Lee EJ, Sung MH, Ahn HK, Kim YA, Ahn HK; Supervision: Sung MH; Writing - original draft: Lee EJ; Writing - review \& editing: Sung $\mathrm{MH}$.

\section{간호대학생이 임상실습에서 경험하는 무례함, 극복력, 사회적 지지가 소진에 미치는 영향}

\author{
이은정 $\odot,{ }^{1}$ 성미혜 $\odot,{ }^{2}$ 안혜경 $\odot, 3$ 김윤아 $\left({ }^{4}\right.$
}

1인제대학교 대학원생

${ }^{2}$ 인제대학교 교수

3인제대학교 대학원생

4가야대학교 조교수

\title{
Effect of Incivility, Resilience, and Social Support Experienced by Nursing Students on Burnout in Clinical Practice
}

\author{
Eun Jung Lee $\mathbb{D}^{,},{ }^{1}$ Mi-Hae Sung ${ }^{\circledR},{ }^{2}$ Hye-Kyong Ahn ${ }^{\circledR},{ }^{3}$ Yun Ah Kim ${ }^{4}{ }^{4}$ \\ 'Graduate Student, Inje University, Gimhae, Korea \\ ${ }^{2}$ Professor, Department of Nursing, Inje University College of Medicine, Institute of Health Science, Busan, Korea \\ ${ }^{3}$ Graduate Student, Inje University, Gimhae, Korea \\ ${ }^{4}$ Assistant Professor, Gaya University, Gimhae, Korea
}

\section{ABSTRACT}

Purpose: The purpose of the study was to determine effect of incivility, resilience, and social support experienced by nursing students on burnout in clinical practice.

Methods: Subjects were 140 nursing students who agreed to participate in this study. Collected data were analyzed using descriptive statistics, t-test, analysis of variance, Pearson's correlation and stepwise multiple regression with SPSS WIN 23.0 program.

Results: Burnout showed significantly positive correlation with incivility but significantly negative correlations with resilience and social support. Factors affecting burnout were satisfaction with major-dissatisfaction, satisfaction with major-average, social support, grade, and relationship with peers. Satisfaction with major (dissatisfaction) had the greatest effect on burnout, explaining $41 \%$ of the total variance.

Conclusion: According to this study, dissatisfaction with major was identified as the most significant factor influencing burnout of nursing students in clinical practice. Therefore, it is important to develop and implement programs that can reduce dissatisfaction with major and increase social support and relationship with peers in order to lower burnout of nursing students. In addition, a systemic management of fourth-grade students with a high level of clinical practice is necessary to reduce the level of clinical practice. The authors declared no conflict of interest.

Keywords: Nursing students; Incivility; Resilience, psychological; Social support; Burnout 주요어: 간호 대학생; 무례함; 극복력; 사회적 지지; 소진 


\section{서론}

\section{1. 연구의 필요성}

간호대학생은 임상실습을 통해 총체적인 간호지식과 실무를 통합할 수 있는 임상수행능력 을 기르며 의사소통능력과 기본 간호술을 적용해 보고 문제해결능력을 익힐 뿐만 아니라 전 문 직업인으로서의 가치체계를 개발할 수 있는 기회를 가짐으로써 전문직 간호사로서의 기 본능력을 갖추게 된다[1]. 최근 정보의 대중화와 의료기술의 발전, 양질의 의료서비스에 대 한 소비자 요구 증가 등 빠르게 변화하고 있는 보건의료 환경으로 인해 임상현장에서는 보 다 전문적이고, 수준 높은 임상수행 능력을 갖추도록 요구하고 있다[2]. 이에 임상실습은 간 호대학생이 교내에서 습득한 이론적 지식을 실제 현장에서 통합적으로 적용해 보면서 간호 사에게 필요한 기술과 태도를 직접 터득하게 되는 교과과정의 핵심적인 내용이라고 볼 수 있 다[3].

간호대학생은 이론수업은 물론 임상실습을 함께 병행해야 하므로 이에 대한 부담이 높고[4], 이러한 상태가 지속되면 피로, 불면, 불안, 우울증이 나타나고 임상실습에 적응하지 못하며 결국 실습과 관련된 소진을 경험하게 된다[5]. 간호대학생은 다른 대학생들에 비해 소진정도 가 높은데[4], 특히 실습 시 경험하는 소진은 이들의 정신건강뿐만 아니라, 학년, 성별, 대학 생활 만족도, 전공 만족도, 임상실습 경험, 임상실습 만족도[6]와 완벽주의 성향, 극복력, 임 상실습교육환경에 대한 인식, 실습동료들과의 관계 등 다양한 요인과 관련되어 있다[5]. 따 라서, 간호대학생이 임상실습 시 경험하게 되는 소진에 영향을 미치는 요인을 구체적으로 파 악할 필요가 있다.

무례함은 대상을 해치려는 의도가 모호한 상호 존중해야 하는 근무지의 규범을 위반하는 낮 은 강도의 일탈된 행동이며, 타인에 대한 존중이 부족함을 드러내는 무례한 행동을 의미하는 것이다[7]. 간호대학생은 임상실습 시 의료인, 환자 및 보호자, 기관관계자 등의 무시나 무관 심, 불쾌한 표정, 생각 없는 행동, 비꼬는 행동, 귀찮아하는 표정 등과 같은 무례한 행동을 경 험하게 되는데, 이러한 무례함이 이들에게 정신적 스트레스를 가중시키며[8], 직업으로 임 상간호사를 선택하는 과정에 부정적인 영향을 미치고, 소진의 원인이 되기도 한다[9]. 그러 나 간호대학생이 임상실습 시 경험하는 무례함과 소진에 관한 연구는 많이 다루어지지 않은 실정이다.

간호대학생은 개인이 가진 신념이나 내적 자원에 따라 자신이 처한 환경을 잘 극복하여 맡 은 임무를 훌륭히 수행해 내기도 하고 정서적 문제나 대학생활에 대한 부적응으로 어려움에 처하기도 한다[10]. 변화하는 상황적 요구나 스트레스가 많은 환경에 처했을 때, 융통성 있 게 반응하여 적응을 할 수 있는 극복력은 새로운 상황에서 오는 긴장 및 인내의 수준을 조절 하고 낮선 상황에 유연하게 반응하게 하여 성공적인 적응이 가능해진다[11]. 이러한 간호대 학생의 극복력은 임상실습 시 다양한 상황에서 직면하게 되는 많은 문제들을 긍정적으로 해 결할 수 있도록 한다[12]. 따라서, 간호대학생의 극복력과 소진 간의 관계를 규명하는 중요하 다고 할 수 있다.

한편, 간호대학생이 임상실습 시 직면하게 되는 상황이나 문제를 해결하는데 자신과 관계를 맺고 있는 사람들로부터의 사회적 지지가 긍정적인 요소가 될 수 있다[13]. 가족과 또래의 인 
정과 지지는 자율성이 발달하고 자아정체감이 형성되는 대학생 시기에 긍정적인 자아개념 형성에 영향을 줌으로써 학업과 취업에 관련된 스트레스를 낮춰주며, 문제 상황에 대한 갈등 을 완화시키고 효과적으로 대처해 나갈 수 있도록 돕는 의미 있는 요소로 작용한다[14]. 이에 간호대학생이 임상실습 시 경험하는 소진과 사회적 지지와의 관계에 대한 규명이 필요하나 아직 이에 대한 연구는 미흡한 실정이다.

지금까지 국내 간호대학생의 임상실습 시 경험하는 소진에 관한 연구를 보면 임상실습 스트 레스 [3,14], 무례함[7,9], 정신건강[6], 감정노동 [15]과 같은 부정적인 측면의 연구가 대부분이 며, 긍정적 측면의 극복력, 사회적 지지와 같은 변수가 소진에 미치는 영향을 파악한 연구는 미흡한 실정으로 이들의 관계를 규명하는 것이 필요하다고 생각된다.

따라서 본 연구는 간호대학생을 대상으로 임상실습에서 경험하는 무례함, 극복력, 사회적 지 지 및 소진 간의 관계를 파악하고, 이들이 소진에 미치는 영향을 규명함으로써, 간호대학생이 임상실습시 경험하는 소진을 예방할 수 있는 효율적인 간호중재전략을 모색하고 나아가간호 대학생의 임상실무능력을 향상시키기 위한 기초자료를 제공하고자 한다.

\section{2. 연구목적}

본 연구는 간호대학생이 임상실습에서 경험하는 무례함, 극복력, 사회적 지지 및 소진 간의 관계를 파악하고, 이들이 임상실습에서 경험하는 소진에 영향을 미치는 요인을 확인하기 위 함이며, 구체적인 목적은 다음과 같다.

- 대상자의 임상실습에서 경험하는 무례함, 극복력, 사회적 지지 및 소진의 정도를 파악 한다.

- 대상자의 일반적인 특성에 따른 소진의 차이를 파악한다.

• 대상자의 제 변수 간의 상관관계를 파악한다.

- 대상자의 소진에 영향을 미치는 요인을 파악한다.

\section{연구방법}

\section{1. 연구설계}

본 연구는 간호대학생의 임상실습에서 경험하는 무례함, 극복력, 사회적 지지와 소진 간의 관계를 파악하고, 소진에 미치는 영향요인을 확인하기 위한 서술적 조사연구이다.

\section{2. 연구대상}

본 연구의 대상자는 부산, 경남에 있는 대학교 중 간호학과에 재학 중이며 임상실습에 참여 하는 3,4 학년 간호대학생을 대상으로 하였다. 연구대상자 수는 G-power 3.1.9.2 program을 이 용하여 산출했을 때, 유의수준 $\alpha$ 는 .05, 검정력(1- $\beta$ ) 0.80, 효과크기 .15 (medium), 예측변수 10 개(극복력, 무례함, 사회적 지지, 성적, 거주형태인 부모동거, 한 부모 동거, 자취, 전공만족 도, 임상실습만족도, 부속실습병원)로 산출한 결과 다중회귀분석에 필요한 표본 수는 118 명 이었다. 탈락률 $20 \%$ 을 고려하여 140 부를 배부하였으나 불성실한 응답이 없어 $100 \%$ 모두 최 종자료 분석에 활용하였다. 


\section{3. 연구도구}

1) 무례함

간호대학생이 간호사와의 관계에서 경험한 무례함을 측정하기 위해 개발한 도구인 The Uncivil Behavior in Clinical Nursing Education [16]을 번역한 도구 [9]를 사용하였다. 도구 사용 전 메일을 통해 원저자와 번역자의 허락을 받은 후 사용하였다. 이 도구는 총 12 개 문항으로 5점 Likert 척 도로 ‘매우 자주’에 4점, ‘자주’에 3점, '가끔’에 2점, ‘드물게’에 1점, ‘전혀 없음’에 0점으로 이루어 졌다. 점수가 높을수록 간호대학생이 경험하는 무례함 정도가 높음을 의미한다. 개발 당시 도 구의 신뢰도는 Cronbach's $\alpha=.88$ 이었고, 본 연구에서는 Cronbach's $\alpha=.89$ 이었다.

\section{2) 극복력}

간호대학생을 대상으로 개발한 회복탄력성 측정한 도구[17]로 사용하였다. 도구 사용 전 메 일을 통해 도구 개발자의 허락을 받은 후 사용하였다. 이 도구는 총 24 개 문항으로 5 점 Likert 척도로 ‘매우 그렇다’에 5점, ‘그렇다’에 4점, ‘보통이다’에 3점, ‘그렇지 않다’에 2점, ‘전혀 그 렇지 않다’에 1점으로 이루어졌다. 부정적 문항에 대해서는 역환산 처리하였고, 점수가 높을 수록 극복력이 높음을 의미한다. 개발 당시 도구의 신뢰도는 Cronbach's $\alpha=.84$ 이었고, 본 연 구에서는 Cronbach's $\alpha=.87$ 이었다.

\section{3) 사회적 지지}

Park [18]이 개발한 사회적 지지 척도 25 문항을 학생에게 적합한 18 문항을 발췌한 도구[19] 를 사용하였다. 도구 사용 전 메일을 통해 도구 개발자 및 수정한 자의 허락을 받은 후 사 용하였다. 이 도구는 총 18 개 문항으로 '아주 많이 그렇다’에 5점, '많이 그렇다’에 4점, ‘보통이다’에 3점, ‘약간 그렇다'에 2점 ‘거의 그렇지 않다’에 1점으로 점수가 높을수록 사회적 지지 정도가 높음을 의미한다. 개발 당시 도구의 신뢰도는 Cronbach's $\alpha=.95$ 이었고, Woo [19] 의 연구에서 Cronbach's $\alpha=.98$ 이었으며, 본 연구에서는 Cronbach's $\alpha=.97$ 이었다.

\section{4) 소진}

일반인의 소진을 측정하기 위해 개발한 말라크 소진척도(Maslach Burnout Inventory-General Survey) [20]를 한국인 학생을 대상으로 수정한 도구[21]를 사용하였다. 도구 사용 전 메일을 통해 도구 개발자 및 수정자의 허락을 받은 후 사용하였다. 이 도구는 총 15 개 문항으로 '매우 그렇다’에 5점, ‘그런 편이다’에 4점, ‘보통이다’에 3점, ‘그렇지 않다’에 2점 ‘전혀 아니다’에 1 점으로 이루어졌다. 하위 범주는 정서적 탈진(1-5번), 냉담(6-9번), 무능감(10-15번)으로 구성 되어 있으며 무능감은 역문항으로 역환산하였으며, 점수가 높을수록 소진의 정도가 높음을 의미한다. 개발 당시 하위도구의 신뢰도는 정서적 탈진 .85, 냉담 .78, 무능감 .73이었으며, 수 정한 도구를 사용한 연구[21]에서의 하위도구 신뢰도는 정서적 탈진 .86, 냉담 .82, 무능감 .82 였다. 본 연구에서는 Cronbach's $\alpha=.81$ 이었다.

\section{4. 자료수집}

본 연구의 목적과 방법, 절차 및 개인 정보 보호에 대해서 I학교 생명윤리위원회의 승인(IRB No. INJE2018-07-004-001)을 받은 후 2018년 8월 27일부터 10월 11일까지 자료수집을 하였다. 자료수집방법은 부산, 경남에 있는 대학교 중 간호학과에 재학 중이며 임상실습에 참여하는 3,4 학년 간호대학생을 대상으로 연구자의 연구의 취지와 목적, 참여의 자율성, 개인 정보 비 밀 유지를 설명 후 연구참여에 동의하여 동의서를 작성한 간호대학생을 대상으로 설문지를 
배부하였다. 구조화된 설문지를 직접 작성하도록 하고 설문지 작성 후 즉시 회수하였으며 연 구에 참여한 모든 대상자에게는 소정의 선물을 제공하였다.

\section{5. 자료분석}

자료분석은 SPSS/WIN 23.0 프로그램(IBM Corp., Armonk, NY, USA)을 이용하여 분석하였으 며, 대상자의 일반적 특성은 빈도와 백분율, 평균과 표준편차로 분석하였다. 대상자의 임상 실습에서 경험한 무례함, 극복력, 사회적 지지 및 소진은 평균과 표준편차로, 대상자의 일반 적 특성에 따른 소진의 차이는 t-test와 analysis of variance로, 사후검증은 Scheffé test로 분석 하였다. 대상자의 제 변수 간의 상관관계는 Pearson's correlation coefficient로 분석하였고, 소 진에 영향을 미치는 요인은 stepwise multiple regression으로 분석하였다.

\section{연구결과}

\section{1. 일반적 특성}

대상자의 성별은 여자가 $94.3 \%$ 이었고, 평균 연령은 22.6세였으며, 학년은 3학년이 59.3\%(83 명)이었다. 종교는 $70.7 \%$ 가 없다고 응답하였고, 거주형태는 부모와 동거가 $62.1 \%$ 이었으 며, 학점은 3.01 이상 4.0 이하가 $75 \%$ 이었다. 입학동기는 $42.9 \%$ 가 ‘졸업 후 취업률이 높아 서'라고 응답하였고, 전공만족도는 ‘만족한다’가 $57.9 \%$ 로 가장 많았다. 임상실습만족도는 '만족한다'가 $50.7 \%$ 이었으며, 실습동료와의 관계는 $86.4 \%$ 가 ‘좋다'로 응답하였다. 실습에 서 어려웠던 관계는 $66.4 \%$ 가 ‘수간호사 및 일반간호사의 관계'로 응답하였으며, $64.3 \%$ 가 ‘실습부속병원이 아니다’라고 응답하였다(Table 1).

\section{2. 대상자의 임상실습에서 경험한 무례함, 극복력, 사회적 지지, 소진정도}

대상자의 임상실습에서 경험한 무례함은 4점 만점에 평균 0.54 (standard deviation [SD] 0.56)점이었고, 극복력은 5점 만점에 평균 3.73 (SD 0.43)점이었다. 사회적 지지는 5점 만점 에 평균 3.25 (SD 0.84)점이었으며, 대상자의 임상실습소진은 5점 만점에 평균 2.81 (SD 0.51) 점이었다(Table 2).

\section{3. 대상자의 일반적 특성에 따른 임상실습소진의 차이}

대상자의 일반적 특성에 따른 소진은 학년 $(\mathrm{t}=-3.41, p=.001)$, 전공만족도 $(\mathrm{F}=25.36, p<.001)$, 임 상실만족도 $(\mathrm{F}=31.24, p<.001)$, 실습동료와의 관계 $(\mathrm{t}=3.83, p<.001)$, 실습에서 어려웠던 관계 ( $\mathrm{F}=3.43, p=.019)$ 에서 통계적으로 유의한 차이가 나타났다(Table 3).

\section{4. 대상자의 임상실습에서 경험한 무례함, 극복력, 사회적 지지, 소진 간의 관계} 대상자의 소진은 극복력 $(\mathrm{r}=-.36, p<.001)$, 사회적 지지 $(\mathrm{r}=-.36, p<.001)$ 와 부적 상관관계가 있 었고, 무례함(r=.23, $p=.008)$ 과 정적 상관관계가 있었다(Table 4).

\section{5. 임상실습소진의 영향요인}

대상자의 임상실습의 소진에 미치는 영향을 파악하기 위해 일반적 특성 중 소진에 유의한 차 이를 나타낸 학년, 전공만족도, 임상실습만족도, 실습동료와의 관계, 실습에서 어려웠던 관 계 등 5 개를 포함하여 무례함, 극복력, 사회적 지지를 독립변수로 투입한 후 다중회귀분석을 


\begin{tabular}{|c|c|c|c|}
\hline Variables & Categories & $\mathrm{n}(\%)$ & $\mathrm{M} \pm \mathrm{SD}$ \\
\hline \multirow[t]{2}{*}{ Sex } & Male & $8(5.7)$ & \\
\hline & Female & $132(94.3)$ & \\
\hline \multirow[t]{3}{*}{ Age (year) } & $\leq 21$ & $63(45.0)$ & $22.6 \pm 3.1$ \\
\hline & $22-23$ & $50(35.7)$ & \\
\hline & $\geq 24$ & $27(19.3)$ & \\
\hline \multirow[t]{2}{*}{ Grade } & Junior & $83(59.3)$ & \\
\hline & Senior & $57(40.7)$ & \\
\hline \multirow[t]{2}{*}{ Religion } & Yes & $41(29.3)$ & \\
\hline & No & 99 (70.7) & \\
\hline \multirow[t]{4}{*}{ Residence } & With parents & $87(62.1)$ & \\
\hline & With single parent & $6(4.3)$ & \\
\hline & With grandparents or relatives & $3(2.1)$ & \\
\hline & Self-catering & $44(31.4)$ & \\
\hline \multirow[t]{3}{*}{ Academic achievement } & $\leq 3.0$ & $22(15.7)$ & \\
\hline & $3.01-4.0$ & $105(75.0)$ & \\
\hline & $\geq 4.01$ & $13(9.3)$ & \\
\hline \multirow[t]{5}{*}{ Motivation for admission } & High school record & $13(9.3)$ & \\
\hline & Recommendation of others & $34(24.3)$ & \\
\hline & Volunteer spirit & $19(13.6)$ & \\
\hline & Employment rate & $60(42.9)$ & \\
\hline & Nurse's good image & $14(10.0)$ & \\
\hline \multirow[t]{3}{*}{ Satisfaction with major } & Dissatisfaction & $15(10.7)$ & \\
\hline & Average & $44(31.4)$ & \\
\hline & Satisfaction & $81(57.9)$ & \\
\hline \multirow[t]{3}{*}{ Satisfaction with clinical practice } & Dissatisfaction & $21(15.0)$ & \\
\hline & Average & $48(34.3)$ & \\
\hline & Satisfaction & $71(50.7)$ & \\
\hline \multirow[t]{2}{*}{ Relationship with peers } & Average & $19(13.6)$ & \\
\hline & Good & $121(86.4)$ & \\
\hline \multirow[t]{4}{*}{ A difficult relationship in practice } & Head nurse or general nurse & $93(66.4)$ & \\
\hline & Someone else (medicine, exam room, etc.) & $11(7.9)$ & \\
\hline & Patients and carers & $25(17.9)$ & \\
\hline & Practical colleague & $11(7.9)$ & \\
\hline \multirow[t]{2}{*}{ Affiliated hospital } & Yes & $50(35.7)$ & \\
\hline & No & $90(64.3)$ & \\
\hline
\end{tabular}

$\mathrm{M} \pm \mathrm{SD}=\mathrm{mean} \pm \mathrm{standard}$ deviation.

Table 2. Degree of Incivility, Resilience, Social Support, Burnout of Clinical Practice in Nursing College Students $(N=140)$

\begin{tabular}{lcc}
\hline Variables & $\mathrm{M} \pm \mathrm{SD}$ & Range (min-max) \\
\hline Resilience & $3.73 \pm 0.43$ & $2.29-5.00$ \\
Incivility & $0.54 \pm 0.56$ & $0.00-2.75$ \\
Social support & $3.25 \pm 0.84$ & $1.22-5.00$ \\
Burnout & $2.81 \pm 0.51$ & $1.60-4.20$ \\
\hline
\end{tabular}

$\mathrm{M} \pm \mathrm{SD}=$ mean \pm standard deviation.

실시하였다. 일반적 특성 중 범주형 항목인 전공만족도(만족기준), 일상실습만족도(만족기 준), 실습에서 어려웠던 관계(실습 동료들과의 관계 기준)은 가변수(Dummy variable)처리하 여 분석하였으며, 분석 전 오차 항들 간에 자기 상관이 있는지 알아보기 위해 Durbin-Watson 통계량을 구한 결과 1.871 로 오차들 간에 자기상관이 없는 것으로 나타났다. 독립변수들 간 의 다중 공선성이 있는지 검증해 보기 위하여 공차한계(Tolerance)와 분산팽창인자(Variance Inflation Factor [VIF])를 확인하였으며, 공차한계는 .88 .94로 모두 .10 이상으로 값이 나타났 고, VIF는 1.14 1.07로 모두 10미만의 값으로 나타나 변수들 간에는 다중 공선성상의 문제가 없는 것으로 확인되었다. 분석결과, 간호대학생의 가장 강력한 예측인자는 전공만족도-불만 족( $\beta=.37, p<.001)$ 이었고, 전공만족도-보통( $\beta=.33, p<.001)$, 사회적 지지 $(\beta=-.26, p<.001)$, 학년 
Table 3. Differences in Burnout of Clinical Practice in Nursing College Students

$(N=140)$

\begin{tabular}{|c|c|c|c|c|}
\hline Variables & Categories & $\mathrm{M} \pm \mathrm{SD}$ & $t / F(p)$ & Scheffé test \\
\hline \multirow[t]{2}{*}{ Sex } & Male & $2.97 \pm 0.37$ & $0.92(.356)$ & \\
\hline & Female & $2.80 \pm 0.51$ & & \\
\hline \multirow[t]{3}{*}{ Age (year) } & $\leq 21$ & $2.77 \pm 0.46$ & $0.34(.715)$ & \\
\hline & $22-23$ & $2.84 \pm 0.52$ & & \\
\hline & $\geq 24$ & $2.83 \pm 0.59$ & & \\
\hline \multirow[t]{2}{*}{ Grade } & Junior & $2.69 \pm 0.49$ & $-3.41(.001)$ & \\
\hline & Senior & $2.98 \pm 0.49$ & & \\
\hline \multirow[t]{2}{*}{ Religion } & Yes & $2.80 \pm 0.52$ & $-0.13(.893)$ & \\
\hline & No & $2.81 \pm 0.50$ & & \\
\hline \multirow[t]{4}{*}{ Residence } & With parents & $2.80 \pm 0.54$ & $0.68(.563)$ & \\
\hline & With single parent & $2.62 \pm 0.50$ & & \\
\hline & With grandparents or relatives & $3.13 \pm 0.77$ & & \\
\hline & Self-catering & $2.82 \pm 0.41$ & & \\
\hline \multirow{3}{*}{$\begin{array}{l}\text { Academic } \\
\text { achievement }\end{array}$} & $\leq 3.0$ & $3.02 \pm 0.52$ & $3.03(.051)$ & \\
\hline & $3.01-4.0$ & $2.78 \pm 0.51$ & & \\
\hline & $\geq 4.01$ & $2.63 \pm 0.32$ & & \\
\hline \multirow{5}{*}{$\begin{array}{l}\text { Motivation for } \\
\text { admission }\end{array}$} & High school record & $2.79 \pm 0.44$ & $1.24(.299)$ & \\
\hline & Recommendation of others & $2.78 \pm 0.57$ & & \\
\hline & Volunteer spirit & $2.78 \pm 0.43$ & & \\
\hline & Employment rate & $2.89 \pm 0.51$ & & \\
\hline & Nurse's good image & $2.57 \pm 0.47$ & & \\
\hline \multirow{3}{*}{$\begin{array}{l}\text { Satisfaction with } \\
\text { major }\end{array}$} & Dissatisfaction $^{a}$ & $3.38 \pm 0.43$ & $25.36(<.001)$ & $a>b>c$ \\
\hline & Average $^{b}$ & $2.98 \pm 0.47$ & & \\
\hline & Satisfaction $^{c}$ & $2.60 \pm 0.42$ & & \\
\hline \multirow{3}{*}{$\begin{array}{l}\text { Satisfaction with } \\
\text { clinical practice }\end{array}$} & Dissatisfaction $^{\mathrm{a}}$ & $3.28 \pm 0.47$ & $31.24(<.001)$ & $a>b>c$ \\
\hline & Average $^{b}$ & $2.99 \pm 0.37$ & & \\
\hline & Satisfaction $^{c}$ & $2.54 \pm 0.44$ & & \\
\hline \multirow{2}{*}{$\begin{array}{l}\text { Relationship with } \\
\text { peers }\end{array}$} & Average & $3.20 \pm 0.54$ & $3.83(<.001)$ & \\
\hline & Good & $2.74 \pm 0.47$ & & \\
\hline \multirow{4}{*}{$\begin{array}{l}\text { A difficult } \\
\text { relationship in } \\
\text { practice }\end{array}$} & Head nurse or general nurse ${ }^{a}$ & $2.83 \pm 0.50$ & $3.43(.019)$ & $d>b$ \\
\hline & Someone else (medicine, exam room, etc.) & $2.42 \pm 0.58$ & & \\
\hline & Patients and carers ${ }^{c}$ & $2.77 \pm 0.42$ & & \\
\hline & Practical colleague $^{d}$ & $3.08 \pm 0.51$ & & \\
\hline \multirow[t]{2}{*}{ Affiliated hospital } & Yes & $2.87 \pm 0.47$ & $1.20(.231)$ & \\
\hline & No & $2.77 \pm 0.52$ & & \\
\hline
\end{tabular}

$\mathrm{M} \pm \mathrm{SD}=\mathrm{mean} \pm \mathrm{standard}$ deviation.

Table 4. Correlation among Incivility, Resilience, Social Support, Burnout of Clinical Practice in Nursing College Students

\begin{tabular}{lcccc}
\hline Variables & \multicolumn{3}{c}{$r(p)$} \\
\cline { 2 - 5 } & Resilience & Incivility & Social support & Burnout \\
\hline Resilience & 1 & & & \\
Incivility & $-.09(.309)$ & 1 & 1 & 1 \\
Social support & $.29(<.001)$ & $-.36(<.001)$ & $-.36(<.001)$ & 1 \\
Burnout & $-.36(<.001)$ & $.23(.008)$ & & \\
\hline
\end{tabular}

( $\beta=.20, p=.004)$, 실습동료와의 관계( $\beta=.17, p=.016)$ 가 영향요인으로 파악되었으며, 모형의 설 명력은 41\%이었다( $\mathrm{F}=19.94, p<.001)$ (Table 5).

\section{논의}

본 연구는 간호대학생을 대상으로 임상실습에서 경험한 무례함, 극복력, 사회적 지지와 소 진 간의 관계를 파악하고, 이들의 소진에 영향을 미치는 요인을 규명함으로써 간호대학생 
Table 5. Factors influencing on Burnout of Clinical Practice in Nursing College Students

\begin{tabular}{|c|c|c|c|c|c|c|c|c|}
\hline Variables & B & SE & $\beta$ & $\mathrm{t}$ & $p$ & Adj. $R^{2}$ & $\mathrm{~F}$ & $p$ \\
\hline Constant & 2.41 & 0.282 & & 8.56 & $<.001$ & & & \\
\hline \multicolumn{9}{|l|}{ Satisfaction with major ${ }^{\dagger}$} \\
\hline Dissatisfaction & 0.61 & 0.114 & .37 & 5.35 & $<.001$ & .15 & & \\
\hline Average & 0.36 & 0.074 & .33 & 4.79 & $<.001$ & .26 & 19.94 & $<.001$ \\
\hline Social support & -0.16 & 0.040 & -.26 & -3.96 & $<.001$ & .34 & & \\
\hline Grade & 0.21 & 0.070 & .20 & 2.95 & .004 & .38 & & \\
\hline Relationship with peers & 0.24 & 0.099 & .17 & 2.45 & .016 & .41 & & \\
\hline
\end{tabular}

$\mathrm{SE}=$ standard error; Adj. $\mathrm{R}^{2}=$ adjusted coefficient of determination $\mathrm{R}^{2}$.

${ }^{\dagger}$ Dummy variable: reference=satisfaction, Durbin-Watson: 1.871, Variance Inflation Factor: 1.14-1.07.

의 임상실습소진을 예방할 수 있는 효율적인 간호중재전략을 모색하는데 도움이 되는 기초 자료를 제공하고자 시도하였다. 본 연구의 결과를 중심으로 다음과 같이 논의하고자 한다.

간호대학생의 무례함의 점수는 4점 만점에 평균 0.54점으로 나타났다. 이는 같은 도구를 사 용하여 3, 4학년 간호대학생을 대상으로 한 연구[22]에서 평균 1.18점으로 나온 결과보다 낮 은 것이나, 두 논문에서 간호대학생의 무례함 점수는 공통적으로 낮음을 알 수 있다. 이러한 결과는 무례함은 언어적 폭력이나 괴롭힘과 같은 가시적인 행동에 한정되지 않고 무시하는 언행이나 표정, 성가신 존재로 여기는 말투나 눈빛 등의 비언어적, 비존중적 행동이 포함되 지만[16], 우리나라의 경우 사회관계가 갖는 특성상 선배나 연장자의 언행에 대해 허용적임 을 볼 때[23], 간호대학생이 간호사의 무례함을 경험하였음에도 낮게 나타난 것으로 판단된 다. 그러나 낮은 정도의 무례함이라 해도 임상실습 중 경험하는 무례함은 소진을 유발하므로 [16], 이에 대한 추후 반복연구가 필요하다고 생각된다.

간호대학생의 극복력 점수는 5 점 만점에 평균 3.73점으로 나타났다. 이는 같은 도구를 사용하 여 3,4학년 간호대학생을 대상으로 한 연구[24]에서 간호대학생의 극복력 점수가 평균 3.56 점으로 나온 결과보다 높은 것이다. 이러한 결과는 본 연구의 대상자 중 간호학 전공만족도 에서 $57.9 \%$ 의 대상자가 만족한다로, 임상실습에 대한 만족도에서 $50.7 \%$ 의 대상자가 만족한 다로 나온 결과와 관련이 있다고 본다. 극복력은 회복탄력성, 회복력, 탄성과 같은 맥락으로 크고 작은 다양한 역경과 시련을 도약의 계기로 삼아 위기 상황을 성공적으로 대처하고 극복 하여 적응하고자 하는 긍정적인 적응력을 의미하는 것으로[25], 개인의 극복력 정도가 신체 적, 정신적 스트레스를 최소화하여 소진을 감소시키는 요인으로 보고되고 있다. 그러므로 간 호대학생의 실습 시 소진을 감소시키기 위하여 긍정적인 임상실습교육환경을 조성하여 임 상실습만족도를 향상시키고 대학교 시기동안 간호학에 대한 만족도 및 대학생활 만족도를 증진시킬 수 있는 중재가 필요하다[5].

한국간호평가원에 의하면 국내 간호교육 임상실습은 1,000 시간 이상이 요구되는 필수교육 과정이다[26]. 대부분의 학생들은 간호교육의 완성을 위해 임상교육을 통한 실제 임상현장 에서 직접 환자를 대면하여 학생이 습득한 이론교육과 간호술기를 적용하며 의사소통 능력, 비판적 사고, 시간관리 기술을 개발하고 간호사로서 업무를 수행할 수 있는 자신감을 증가 시키는 계기가 된다[27]. 따라서 간호대학생들의 임상실습 중 경험하는 소진 정도를 낮추고 임상실무 적응능력을 향상시킬 수 역량강화 특강 프로그램 등의 교양 강좌 개설의 마련이 필 요할 것으로 생각된다. 
간호대학생의 사회적 지지의 점수는 5점 만점에 평균 3.25점으로 나타났다. 이는 같은 도구 를 사용하여 부산, 경남, 경북지역 3,4학년 간호대학생 560명을 대상으로 한 연구[19]에서 간 호대학생의 사회적 지지 점수가 평균 2.81점으로 나온 결과보다 높은 것이다. 이러한 결과는 대상자의 $62.1 \%$ 가 부모와 동거하고, 대상자의 $86.4 \%$ 가 실습동료들과의 관계가 좋다고 응답 한 결과와 관련이 있다고 생각한다. 개인이 사회적 지지를 지각하는 정도가 높을수록 심리적 안녕감이 증가하고, 스트레스로 인한 고통이 감소하며, 스트레스를 인식하는 심각성도 감소 한다는 연구[28] 결과에서 알 수 있듯이 사회적 지지를 많이 받게 하고 많이 받고 있다고 인 식하게 해 주는 것이 중요하다고 할 수 있다. 따라서 간호대학생 개인이 갖고 있는 존재의 가 치와 인식을 높이고 개인의 관계망 구성과 정서의 표현, 격려, 이해 등의 원활한 감정 교류와 상담을 위한 학내 정규 상담시간을 마련하고 솔직한 임상실습의 조언이나 충고를 위한 병원 내 간호대학생과 실습병동 간호사간의 프리셉터 제도를 좀 더 적극적으로 활성화할 필요하 다고 생각된다.

간호대학생의 소진의 점수는 5점 만점에 평균 2.81점으로 나타났다. 이는 같은 도구를사용하여 3 , 4 학년 간호대학생을 대상으로 한 연구[5]에서간호대학생의 소진 점수가 평균 2.92점으로 나온 결 과와 유사한 것이다. 이러한 결과는 대상자의 $42.9 \%$ 가간호학과 입학동기가 졸업 후 취업률이 높 아서였고, 대상자의 $64.3 \%$ 가 실습지가 부속병원이 아니라고 응답한 결과와 관련이 있다고 생각 한다. 실습부속병원이 없는 경우 소진정도가 높은 것은 안정적인 실습을 위한 주요 실습지가 부 재하여 여러 병원의 낮선 환경에서 실습하기 때문인 것으로[6], 실습부속병원이 없는 경우간호 대학생의 소진 정도가 더 높게 나온 연구[5] 결과가 이를 뒷받침 해 주고 있다. 따라서 임상실습지 는 교육기관에 비해매우 부족하여 대부분 여러 기관에 임상실습교육을 의뢰하는 현실이므로 각 기 다른 여러병원에서의 낯선 병원 환경에서 오는 소진을 예방하기 위해지도교수와꾸준한상담 시간, 정서적 지지와격려가 필요하고효과적인 대처전략이 필요하다[5].

간호대학생의 일반적 특성에 따른 소진은 학년, 전공만족도, 임상실습 만족도, 실습동료들 과의 관계, 실습에서 어려웠던 관계에서 통계적으로 유의한 차이가 있었다. 이는 같은 도구 를 사용하여 3, 4학년 간호대학생을 대상으로 한 연구 [5]에서 학년, 전공만족도, 임상실습 만 족도에서 유의한 차이를 보인 결과와 부분적으로 일치하는 것이다. 이러한 결과는 4학년 간 호대학생의 소진 정도가 높게 나타난 연구 $[6,9,12]$ 가 이를 뒷받침 해 주며, 이는 4학년 간호대 학생은 수술실, 응급실, 중환자실, 소아과 등 특수부서의 임상실습을 경험하며 좀 더 심화과 정의 실습환경과 국가고시를 비롯한 학업의 부담감, 취업과 진로의 문제 등 여러 복합적인 문제로 인해 소진정도가 더 높게 나타났다고 생각해 볼 수 있다. 따라서 실습 전 임상실습 환 경에 대한 정보를 사전에 제공하여 미리 계획되고 철저하게 준비된 오리엔테이션과 실습지 도 및 개인별 상담을 강화하여 정신적.신체적인 소진을 적극적으로 낮출 수 있는 여건을 마 련해 줄 필요가 있다.

간호대학생의 임상실습 중 경험하는 무례함, 극복력, 사회적 지지와 소진 간의 상관관계에 서 소진은 극복력, 사회적 지지와 부적 상관관계가 있었고, 무례함과 정적 상관관계가 있는 것으로 나타났다. 이는 극복력과 소진이 부적 상관관계가 있는 것으로 나타난 연구결과[5], 사회적 지지와 소진이 부적상관관계가 있는 것으로 나타난 연구결과[29]와 무례함과 소진이 정적상관관계를 나타낸 연구결과[12,22]와 일치하는 것이다. 이러한 결과를 볼 때 간호대학 생의 소진을 예방하고 감소시키기 위해서는 이들의 극복력과 사회적 지지 체계를 높일 수 있 
는 방법을 모색하고 임상실습환경에서 나타날 수 있는 간호사의 무례함을 낮출 수 있는 교육 프로그램을 개발하여 교육하는 중재방안이 필요할 것으로 생각된다.

간호대학생의 소진의 영향요인은 전공만족도, 사회적 지지, 학년, 실습동료와의 관계이었으 며 모형의 $41 \%$ 이었다. 이는 전공만족도가 임상실습소진의 영향요인으로 나타난 연구 $[5,6]$ 와 일치하는 것으로, 이러한 결과는 대상자의 $42.9 \%$ 가 간호학과 입학동기가 졸업 후 취업률이 높 아서라고 응답하였고, 전공만족도에 불만족 $10.7 \%$, 보통이다 $31.4 \%$ 로 나타난 결과와 관련이 있다고 생각된다. 자신의 적성과 가치관을 고려하여 학과선택을 하기 보다 향후 갖게 될 직업 을 고려하는 이유로 이들에게 입학 후 학교 생활에 잘 적응하여 올바른 간호전문직관을 확립 하고 성장 해 갈 수 있도록 교수-학생간의 친밀감을 높이고, 전공에 대한 긍정적 사고와 개념 형성을 위해 관심을 갖고 지도해야 할 필요가 있다. 또한 가족, 친구, 교수, 실습동료 등과의 사 회적 지지 체계와 실습동료들과의 관계를 향상시켜줌으로써 사회적.심리적.신체적 안녕을 증진시키고 특히 소진 정도가 높게 나타난 4 학년 간호대학생의 소진 정도를 감소시킬 수 있 도록 이들에 대한 각별한 관심과 체계적인 관리가 필요하다[5]. 그러나, 간호대학생의 소진은 극복력과 부적 상관관계가 있었으나, 소진에는 유의하게 영향을 주지 않는 것으로 나타났다. 이러한 결과는 본 연구에서 간호대학생의 전공만족도와 동료와의 관계가 매우 높았고 $60 \%$ 이 상의 학생이 부속병원이 아닌 타 기관에서 실습을 한 것과 관련이 있다고 본다. 임상실습은 자 신이 습득한 지식과 간호술기를 적용하며 의사소통술을 개발하고 간호사로서 업무를 수행할 수 있는 자신감을 증가시키는 계기가 되지만[27], 실습부속병원이 없는 경우 간호대학생의 소 진 정도가 더 높게 나온 연구결과[5]를 볼 때, 본 연구에서 간호대학생의 소진은 타 기관에서의 실습환경으로 이들 개인이 지닌 극복력보다는 전공에 대한 만족도와 동료들과의 관계에 영향 을 받은 것으로 사료된다. 또한, 간호대학생의 소진은 이들이 임상실습 중 경험하는 무례함과 정적 상관관계가 있었으나 소진에는 유의하게 영향을 주지 않았다. 이는 본 연구결과에서 간 호대학생의 무례함 정도가 매우 낮았으며, 소진과도 약한 상관관계를 보인 것과 관련이 있다 고 사료된다. 우리나라의 경우 사회관계가 갖는 특성상 선배나 연장자의 언행에 대해 허용적 이며 [23], 간호대학생이 임상실습시 경험하는 무례함이 낮아 이들의 소진에까지 영향을 미치 지 않은 것으로 보인다. 그러나, 간호대학생을 대상으로 극복력과 무례함과 같은 요인이 소진 에 미치는 영향을 규명하는 연구가 미흡한 실정에서 나온 결과이므로, 이에 대한 명확한 규명 을 위해 반복 연구가 요구된다.

한편, 본 연구는 부산·경남 4 개 대학에 소재하는 간호대학생만을 편의표집 하였으므로 연구 결과를 일반화시키는데 제한점이 있다.

\section{결론}

본 연구는 간호대학생을 대상으로 임상실습 중에 경험하는 무례함, 극복력, 사회적 지지와 소진 간의 관계를 파악하고, 간호대학생의 소진에 영향을 미치는 요인을 확인하기 위해 시 도하였다.

본 연구에서 간호대학생의 소진에 영향을 미치는 요인은 전공만족도-불만족, 전공만족도보통, 사회적 지지, 학년, 실습동료와의 관계로, 이들 변인들에 의한 설명력은 $41 \%$ 였다. 이러 
한 결과를 바탕으로 향후 간호대학생의 소진을 예방하기 위해서는 전공에 대한 불만족 정도 를 줄여주고, 이들의 사회적 지지 체계와 실습동료들과의 관계를 향상시켜줌으로써 전공 만 족도를 향상시킬 필요가 있다. 또한, 4학년 간호대학생의 소진 정도가 높게 나타났으므로 이 들의 소진을 감소시킬 수 있도록 체계적인 관리가 필요함을 알 수 있었다.

본 연구결과를 토대로 간호실무 측면에서 간호대학생의 소진에 영향을 주는 요인을 근거로 이들의 소진을 예방하고감소시키기 위한 프로그램 마련이 필요하다. 간호 연구 및 교육에서 간호대학생을 대상으로 이들의 극복력과 사회적 지지에 관한 연구는 물론 간호대학생이 임 상실습환경에서 성공적으로 대처하고 극복할 수 있는 힘을 기르고 사회적 지지 체계를 구축 할 수 있는 기반을 마련하여야 할 것이다. 간호대학생의 임상실습의 경험은 궁극적으로 병원 및 간호사의 이미지에 영향을 주어 이들의 임상경력에 부정적으로 작용할 것으로 생각되어 추후 임상실습병원과 학교가 산학으로 실습환경 개선을 위한 연구를 제언한다.

\section{REFERENCES}

1. Casey T, Wilson-Evered E. Predicting uptake of technology innovations in online family dispute resolution services: an application and extension of the UTAUT. Computers in Human Behavior. 2012;28(6):2034-2045. CROSSREF

2. Kang YH, Hong MJ. Influencing factors of learning style, critical thinking disposition on clinical performance competency of nursing students. Journal of the Korean Data Analysis Society. 2014;16(5):2841-2853.

3. Lim YJ. The impact of nursing students' emotional labor and clinical practice stress on burnout. Journal of Learner-Centered Curriculum and Instruction. 2017;17(6):461-474. CROSSREF

4. Fang DZ, Young CB, Golshan S, Moutier C, Zisook S. Burnout in premedical undergraduate students. Academic Psychiatry. 2012;36(1):11-16. PUBMED | CROSSREF

5. Cho HH, Kang JM. Factors influencing clinical practice burnout in student nurses. Child Health Nursing Research. 2017;23(2):199-206. CROSSREF

6. Cho HH, Kang JM. Effect of resilience, coping, and mental health on burnout of student nurses. Child Health Nursing Research. 2018;24(2):199-207. CROSSREF

7. Anderson LM, Pearson CM. Tit for tat? The spiraling effect of incivility in the workplace. Academy of Management Review. 1999;24(3):452-471. CROSSREF

8. Kim SL, Lee JE. Relationship among stress, coping strategies, and self-esteem in nursing students taking clinical experience. Journal of Korean Academic Society of Nursing Education. 2005;11(1):98-106.

9. Hong Y, Kim Y, Son H. Effect of nurses' incivility experienced by nursing student, coping on burnout in clinical practice. Journal of Korean Academy of Nursing Administration. 2016;29(4):323-331. CROSSREF

10. Por J, Barriball L, Fitzpatrick J, Roberts J. Emotional intelligence: its relationship to stress, coping, well-being and professional performance in nursing students. Nurse Education Today 2011;31(8):855-860. PUBMED | CROSSREF

11. Seo JH. Effects of nursing image, satisfaction in major, ego-resilience on nursing professionalism of nursing students [master's thesis]. Chungnam: Kongju National University; 2018. 49 p.

12. Hong HH, Kong JH, Kang HS, Jeong HS, Yang SK. The study on the professional self-concept, ego-resilience, clinical competence of nursing students. Journal of Korean Clinical Health Science. 2014;2(2):98-106. CROSSREF

13. Cohen S, Hoberman HM. Positive events and social supports as buffers of life change stress. Journal of Applied Social Psychology. 1983;13(2):99-125. CROSSREF 
14. Kim BK. Factors related to interpersonal relationship stress in clinical practice among nursing students [master's thesis]. Seoul: Yonsei University; 2017. 87 p.

15. Kang SY. Impact of nursing students' emotional labor on burnout during nursing practice in a hospital: moderating effect of emotional intelligence. Journal of Korean Academy of Nursing Administration. 2015;21(1):77-87.

CROSSREF

16. Anthony M, Yastik J, MacDonald DA, Marshall KA. Development and validation of a tool to measure incivility in clinical nursing education. Journal of Professional Nursing. 2014;30(1):48-55. PUBMED | CROSSREF

17. Yang YH, Kim EM, Yu M, Park SM, Lee HY. Development of the resilience scale for korean nursing college students. Korean Journal of Adult Nursing. 2015;27(3):337-346. CROSSREF

18. Park JW. A study to development a scale of social support [dissertation]. Seoul: Yonsei University; 1985. 127 p.

19. Woo JA. Emotional responses, coping and social support for verbal abuse, sexual harassment, physical threats experienced by nursing students in clinical training [dissertation]. Pusan: Pusan National University; 2017. $153 \mathrm{p}$.

20. Schaufeli WB, Martinez IM, Pinto AM, Salanova M, Bakker AB. Burnout and engagement in university students: a cross-national study. Journal of Cross-Cultural Psychology. 2002;33(5):464-481. CROSSREF

21. Lee J, Puig A, Kim YB, Shin HJ, Lee JH, Lee SM. Academic burnout profiles in Korean adolescents. Stress and Health. 2010;26(5):404-416. CROSSREF

22. Kim JG, Yoo JH, Cheon EY. Realtionship among incivility, burnout, coping and satisfaction to clinical practice experienced by nursing college students in clinical practice. Journal of the Korea Academia-Industrial Cooperation Society. 2017;18(1):316-324. CROSSREF

23. Park JW, Ha NS. Nursing student's clinical experience. Journal of Korean Academy of Psychiatric and Mental Health Nursing. 2003;12(1):27-35.

24. Lee YE, Jang YN. Effects of exposure to violence during clinical practicum, self-esteem and resilience on depression among nursing students. Journal of the Korea Contents Association. 2018;18(8):646-657. CROSSREF

25. Lee YE, Kim EY, Park SY. Effect of self-esteem, emotional intelligence and psychological well-being on resilience in nursing students. Child Health Nursing Research. 2017;23(3):385-393. CROSSREF

26. Korean Accreditation Board of Nursing Education. A report on evaluation of nursing school, 2016. Seoul: Korean Accreditation Board of Nursing Education; 2017.

27. Löfmark A, Wikblad K. Facilitating and obstructing factors for development of learning in clinical practice: a student perspective. Journal of Advanced Nursing. 2001;34(1):43-50. PUBMED | CROSSREF

28. Jeon SY. The relationship between social support, health status, college adjustment and academic achievement in college students. Journal of Korean Society for School \& Community Health Education. 2010;11(1):99-115.

29. Noh YG. Influence of social support on the relationship between practice stress, emotional labor and burnout among nursing students. Journal of Korean Academy of Nursing Administration. 2017;23(5):461-470. CROSSREF 


\section{SUMMARY STATEMENT}

\section{-What is already known about this topic?}

Burnout of nursing students is higher than that of other university students.

- What this paper adds?

This paper revealed that burnout were satisfaction with major-dissatisfaction, satisfaction with major-average, social support, grade, and relationship with peers.

\section{- Implications for practice, education and/or policy}

There is a need to develop and implement programs that can reduce dissatisfaction with major and increase social support and relationship with peers in order to lower burnout level of nursing students. 